\title{
The Dissolved Organic Carbon CONTROVERSY: AN UPDATE
}

\author{
By Jonathan H. Sharp
}

\begin{abstract}
$\mathrm{T}$ HIS NOTE IS INTENDED as an update on activities to improve the measurement of dissolved organic carbon (DOC) in seawater. The exciting and controversial paper by Sugimura and Suzuki (1988) led to renewed interest in DOC in the ocean. It was followed by several early comments in this forum (Jackson, 1988; Williams and Druffel, 1988) and considerable research activity culminating in the comment by Williams (1992) about the Seattle DOC workshop. A historical perspective on DOC measurement is presented here along with comments on publications since the Seattle workshop, highlights of two efforts launched to start the process of interlaboratory comparison for uniform DOC measurements, and an announcement for the next comparison activity. The results referenced here are the product of a cooperative effort by a number of analysts with valuable guidance from a steering committee (see Acknowledgements). The opinions and conclusions are mine.
\end{abstract}

\section{Introduction}

The controversy over the concentration of dissolved organic matter in seawater has a long history, specifically concerning the analytical methods employed in the measurement of DOC. To date, the two most prominent groups of methods for DOC analysis are wet chemical oxidation (WCO) and high temperature combustion (HTC). The most successful WCO methods are persulfate oxidation, ultraviolet irradiation, and a combination of the two. HTC methods were first developed in the former Soviet Union in the 1950s (Skopintsev et al., 1966). With the development of a persulfate WCO method (Menzel and Vacarro, 1964), then considered rapid and easy, indirect comparisons gave rise to the suggestion that the higher values observed by the Soviet HTC method were due, in part, to contamination problems. Several HTC methods developed in Canada in the late

J.H. Sharp, College of Marine Studies, University of Delaware, Lewes, DE 19958, USA 1960s and early 1970s (Gordon and Sutcliffe, 1973; Sharp, 1973; McKinnon, 1978) were also shown by direct or indirect comparisons to give higher DOC values than the WCO methods. These studies were relegated to the realm of "contamination" by some evaluations (e.g., Gershey et al., 1979). More recent modifications of the HTC method by the Japanese (Sugimura and Suzuki, 1988), which resulted in significantly higher DOC values, have rekindled interest in this methodology. This and all recent HTC methods include some form of catalysis, usually platinum. So the questions remain after 40 years: do HTC methods really measure more DOC than WCO methods, can HTC methods give reliable and consistent results, and do all HTC (or all WCO) methods measure the same thing?

Most of the DOC comparisons between these two types of methods have been indirect, based on comparing results from the literature (e.g., Gershey et al., 1979). Also most have been based on the original persulfate oxidation procedure (Menzel and Vacarro, 1964). The DOC distribution typically obtained by this "traditional" method is of low surface water concentrations of $<100 \mu \mathrm{M} \mathrm{C}$ and a flat deep water profile somewhere in the $30-50 \mu \mathrm{M} \mathrm{C}$ range. A modification of the traditional persulfate method removing the inorganic carbon before exposing the sample to persulfate (Sharp, 1973), gave values $\sim 20 \%$ higher than the original method and less flat depth profiles. Although this modification has been adopted in many laboratories, the picture derived from the original Menzel and Vacarro method is still the one most often used in comparisons. The new HTC method (Sugimura and Suzuki, 1988; Suzuki et al., 1985) showed surface values of DOC much higher than traditional analyses (as much as $3-5 \times$ ) and showed a tight inverse correlation with apparent exygen utilization (AOU) in the subsurface Pacific Ocean. Several commercial HTC analyzers are now being used in oceanographic laboratories with results that have been equivocal in some cases.
Do high temperature

combustion methods

really measure more

DOC than wet chemi-

cal oxidation methods? 
The results of the Seattle workshop have been published as a special issue of Marine Chemistry (Volume 41, Number 1-3, 1993). The special issue contains several background papers that directly compare HTC methods to the persulfate or UV oxidation methods. In some of the papers, the differences between methods are not as large as the indirect comparisons had suggested earlier (e.g., Benner and Hedges. 1993). In several of the papers, the importance of the instrument blank is demonstrated (e.g.. Benner and Strom. 1993) and information on how to determine the blank is discussed. In some of the papers with direct comparisons. careful uniform blank corrections for the HTC method are still lacking (Sharp et al.. 1993). The result from the Seattle meeting that affects many in the oceanographic community was the comparison of 34 analyses by workshop participants of four reference natural water samples (Hedges et al., 1993). In that comparison, the full ranges of measured DOC concentrations span an order of magnitude with standard deviations of $>50 \%$ of the mean value. The results are discussed further below. It must be recognized that the analytical procedures used were highly variable, some analysts used methods that were not well established, and some made blank corrections where others did not. In the working subgroups at the Seattle meeting, many of these problems were recognized. It was generally agreed that the assessment of the blank was crucial for future comparisons. Close examination of the extent and variation of HTC blanks (Benner and Strom, 1993) gives emphasis to the possibility that differences between methods may be partially due to errors in blank corrections. With attention to proper blank correction and consideration of differences between various published WCO methods, the differences between older and modern methods may not be as large as has been suggested (Sugimura and Suzuki, 1988: Druffel $e t$ al., 1989).

In a paper added during preparation of the Seattle Workshop report. Suzuki (1993) has retracted his conclusions from the two earlier papers on dissolved organic nitrogen (DON) and DOC (Suzuki et al., 1985; Sugimura and Suzuki. 1988. respectively). This action was taken after careful re-evaluation of the raw data on which the papers were based and recognition of inadequate blank subtraction and $\mathrm{CO}_{2}$ peak measurement. This retraction does not diminish the impact and importance of the two papers; they have created great interest in the field of dissolved organic matter in both seawater and fresh water systems. Perhaps with accurate DOC measurements, it will be possible to evaluate the small real changes that occur in aquatic systems and that are probably important in the global carbon budget.

\section{Published Works Since Seattle}

Two recent studies using an HTC analyzer have been published showing surface ocean DOC values considerably lower than those of Suzuki (Benner et al., 1992; Ogawa and Ogura, 1992). In addition, recent HTC analyses of DOC compared with apparent oxygen utilization show minimal, and not stoichiometrically balanced, correlations in the Atlantic and Pacific oceans (Martin and Fitzwater, 1992; Tanoue, 1992). These recent studies show slightly different DOC levels. Another recent publication shows an inverse correlation between DOC and AOU but somewhat inconsistent and with slopes lower than those predicted by the Redfield ratio (Kepkay and Wells, 1992). Since some of these papers do not indicate sufficient attention paid to blank levels and variability, the absolute values reported may be considered questionable.

Recent papers that provide comparisons of HTC methods to WCO methods show either small or no differences between methods (Hedges and Bergamaschi, 1992; Ogawa and Ogura, 1992). In a comparison of HTC analyses to persulfate oxidation and to $\mathrm{UV}$ oxidation of algal $\mathrm{DO}^{14} \mathrm{C}$, it was found that both of the WCO methods missed $\sim 10-20 \%$ of the DOC (Ridal. 1992). Another recent comparison of the UV oxidation method to $\mathrm{HTC}$ also found $20 \%$ higher yields in oceanic DOC with the HTC method (Chen, 1992). In a recent fresh water comparison, the HTC method showed $\sim 5-10 \%$ higher yield than persulfate oxidation (Kaplan, 1992). None of these recent studies, which do attempt correction for HTC instrument blanks, indicates the 3-5 times higher yield of the HTC method suggested by Sugimura and Suzuki (1988). Two recent papers (Bauer et al., 1992. Druffel et al., 1992) do show higher values by HTC than by UV. However, the HTC data in those papers were previously reported in Druffel et al. (1989) and are analyses done earlier by Suzuki with uncertain blank correction. Therefore, they do not constitute new analyses of DOC.

Only one of these recent publications was interpreted as verifying the high concentrations reported previously by Sugimura and Suzuki (1988) and others were interpreted as failing to do so. Approximate average values based on HTC analysis from some of these recent publications are shown in Table 1. The data show surface oceanic values distinctly lower than those in Sugimura and Suzuki (1988) and oxygen minimum layer values somewhat higher. There is less agreement on the deep water values. However, the publications of the past several months do not show the large surface and immediate subsurface bulge ("new DOC") of Sugimura and Suzuki. These publications also fairly well discount the close inverse correlation of DOC with AOU that has been the "oceanographic consistency" argument for the 
"new DOC" in the surface and immediate subsurface waters (Williams and Druffel, 1988).

\section{Efforts Toward an Analytical Resolution}

As an outgrowth of the Seattle meeting, an effort has been launched to assess better the real differences in methods for both DOC and DON. I am heading this effort with advice from a steering committee consisting of John Hedges, Cindy Lee, Chuck Hopkinson, and Tony Knap. Since several researchers would be doing routine measurements on U.S. JGOFS equatorial Pacific cruises (EqPac), it was also imperative to work with these people to resolve the analytical problems.

A first effort at better analytical consistency was discussion and intercalibration between several of the U.S. JGOFS investigators (E.T. Peltzer, H.W. Ducklow, R. Benner, and J.H. Martin) before the departure in January 1992 of the first EqPac cruises. All were using either homemade or commercial HTC instruments with Pt catalyst. A protocol was developed for collection and analysis of samples for DOC. Experiments were also made using distilled water blanks with very close to zero carbon content. Distilled water was further purified by UV oxidation in the presence of $\mathrm{H}_{2} \mathrm{O}_{2}$ (Peltzer and Brewer, 1993). This blank water was determined by sealed tube combustion (Fry et al., 1993) to have essentially zero carbon content (B. Fry, personal communication). The blank water was then used with two HTC analyzers to subtract the instrument blank. With this blank correction, the two HTC instruments and the sealed tube combustion gave essentially identical values when tested with a sample of aged sea water and with a freshly collected sample from a surface salt pond. The information from this blank correction and the sampling protocol were used during the spring and fall EqPac cruises by analysts involved with the U.S. JGOFS effort, and samples for further comparison activities were collected during the spring cruise using the protocol.

\section{The Bermuda Instrument Comparison}

In May 1992, an interlaboratory comparison was carried out in Bermuda. The focus of that exercise was the commercial HTC instruments that are being used in a number of U.S. and European laboratories. An attempt was made to evaluate behavior of the different instruments rather than to compare the abilities of different laboratories to do the analyses. Results of the Bermuda experiment are in press. (Sharp et al., 1994).

Commercial instruments manufactured by Shimadzu, Dohrmann, and Ionics were used for this exercise. All instruments were being used routinely by researchers involved in the U.S. JGOFS effort and the analysts operated their own instruments in Bermuda for the exercise. Analysis was also done using the sealed ampoule persulfate

Table I

Comparison of oceanic DOC values based on HTC analyses from recent efforts and those of Sugimura and Suzuki.

\begin{tabular}{lcccl}
\hline & \multicolumn{3}{c}{ DOC values, $\mu \mathrm{M} \mathrm{C}$} & \\
\cline { 2 - 4 } \multicolumn{1}{c}{ Location } & Surface & Ox Min & Deep & \multicolumn{1}{c}{ Reference } \\
\hline N. Pacific & $180-320$ & $20-50$ & 100 & Sugimura and Suzuki. 1988 \\
N. Pacific & 82 & 38 & 41 & Benner et al., 1992 \\
N. Atlantic & 150 & 125 & 140 & Kepkay and Wells, 1992 \\
N. Pacific & 85 & & & Ogawa and Ogura, 1992 \\
N. Atlantic & 145 & 115 & 115 & Martin and Fitzwater. 1992 \\
Equatorial Pac & 145 & 115 & 115 & Martin and Fitzwater. 1992 \\
N. Pacific & 150 & 100 & 105 & Tanoue, 1992 \\
Sargasso Sea & 70 & 44 & 44 & Sharp et al., 1994 \\
Equatorial Pac & 72 & 42 & 41 & Sharp et al., (in prep. see text) \\
Sargasso Sea & 60 & 55 & 50 & Menzel and Ryther, 1970 \\
\hline
\end{tabular}

Numbers shown are the approximate value or the rough median value for waters from the surface, oxygen minimum zone, and the deep ocean. The three last lines show the results of the two recent DOC comparison exercises and of the traditional wet chemical method.

method. Since it was apparent that the blank determination was critical (Benner and Strom, 1993) care was taken to address this aspect uniformly. The UV/peroxide method was used to prepare the low carbon water (LCW) blank and a single set of standards was made and used by each analyst. With this approach, good agreement was reached between four analyses on samples from a depth profile in the Sargasso Sea (Fig. 1). Agreement between methods was not perfect but was within about $\pm 15 \%$; i.e., $\sim 4-9 \mu \mathrm{M} \mathrm{C}$ (mean \pm SD) for an individual sample. Details and information on causes of discrepancies are discussed in (Sharp et al., 1994).

\section{The EqPac Exercise}

An interlaboratory comparison consisted of U.S. JGOFS investigators comparing DOC analyses performed in their home laboratories on a set of samples collected during the spring EqPac cruises.

Replicate sets of samples were collected by Ed Peltzer and Hugh Ducklow on two legs of the EqPac spring cruise on the R.V. Thompson. Samples were collected and drawn into bottles cleaned according to the established protocol (see above). They were then quick-frozen and stored until the Thompson returned to Seattle. The samples were then sent to me, relabeled, and distributed to the analysts. In this fashion, none of the analysts knew the exact origin of the individual samples. Blank water (LCW) and two reference standards were sent with the samples. Analysts sent all raw data to me with comments on analytical procedures and any difficulties encountered. I performed data analyses, discussed results with individual analysts, and then shared results with all analysts. Uniform data treatment was used; final data analysis is now being completed, and the manuscript is in preparation.
. . good agreement

was reached between

four analyses on sam-

ples from a depth

profile in the Sargasso

Sea. 


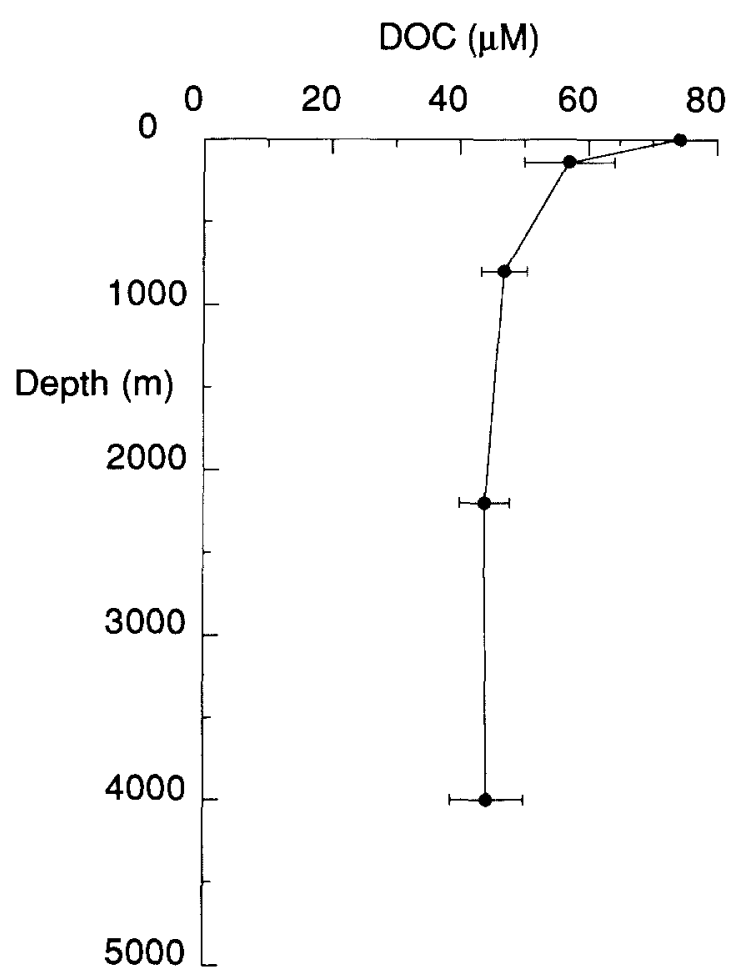

Fig. 1: A depth profile from the Bermuda instrument comparison. Each sample point is the average of 4 independent analyses by 3 HTC instruments and the persulfate oxidation method; brackets around the points are $\pm S D$.

This exercise was also successful in getting good agreement on DOC content of samples. In this case, at least 20 samples from Pacific Ocean depth profiles were compared among six analysts with reference to analyses of these same samples done at sea. An additional 20-29 samples were also run by most of the analysts. The HTC instruments included a homemade one and those manufactured by Shimadzu, Dohrmann, and Ionics. The persulfate WCO method was also used. In general, surface samples had 65-80 $\mu \mathrm{M} \mathrm{C}$ concentration, and deep samples were $\sim 40 \mu \mathrm{M} \mathrm{C}$. An example of the good agreement is shown with a depth profile of averages by the multiple methods (Fig. 2). Since not all of the analysts had all of the samples, only four sets of data were available for this group of samples. The error bars in the figure represent one standard deviation of the replicate analyses of single samples. The standard deviations range from $\pm 0.6 \mu \mathrm{M} \mathrm{C}$, which is as good as the best precision for any one method, to $\pm 6.7 \mu \mathrm{M} \mathrm{C}$.

Another way of showing the agreement of analyses is by comparing them to the results of the Seattle Workshop, using 34 analyses from the Seattle study and five from the EqPac exercise (Fig. 3). Not all of the samples showed good agreement; there are still problems to be resolved (J.H. Sharp, R. Benner, L. Bennett, C.A. Carlson,
S.E. Fitzwater, E.T. Peltzer, and L. Tupas unpublished observations, manuscript in preparation). Also, it should be recognized that the Seattle Workshop data set is marred by the fact that some of the analysts were using methods that were not well established and that some attempted blank corrections while others did not. The point to be made is that with well established methods in the laboratory of an experienced analyst using uniform blank and reference standards, good agreement can be achieved. Now, we need to set criteria to achieve such results routinely.

\section{Current Thoughts}

The results reported here are based on very careful blank correction and have good agreement by multiple instruments. Data from the two exercises are also shown in Table 1 along with some traditional WCO data. In both the Bermuda and EqPac exercises, there was fairly good agreement with the data of Benner et al. (1992) for all three depth zones, but values were somewhat lower than in other recent papers cited here. The fact that these two exercises represent water from both the Atlantic and Pacific oceans and these show very little difference from each other in the deep ocean, discounts arguments that differences may be due to different values found in different areas of the deep ocean. Further analyses with uniform blank evaluation are needed (see below).

The two method comparisons reported here do not show significant differences between the HTC methods used and the modified persulfate oxidation method. Since all the analysts used the same blank to correct for instrument or reagent contamination and used uniform reference standards, the direct comparison is very thorough. It is still possible that the persulfate method measures slightly less than the HTC methods, but the difference would have to be smaller than the scatter reported here, which was in the 10-15\% range. It is possible that other WCO methods may measure less than HTC methods. This question will be addressed with the next interlaboratory comparison.

Since this study and other recent analyses do not agree with the very high values reported by Suzuki, and considering that the latest data show low deep ocean values, it might be argued that data derived by the traditional WCO method are correct. A depth profile in the Sargasso Sea using the traditional method (data from Menzel and Ryther, 1970; Table 1) shows surface values that are lower than the latest analyses and deep water values that are considerably higher. Therefore, it is possible that the most recent analyses do indeed indicate more variation with depth than shown by traditional methods and that the "new DOC" may be important even though not as large as was suggested by Sugimura and Suzuki (1988). 
DOC $(\mu \mathrm{M})$

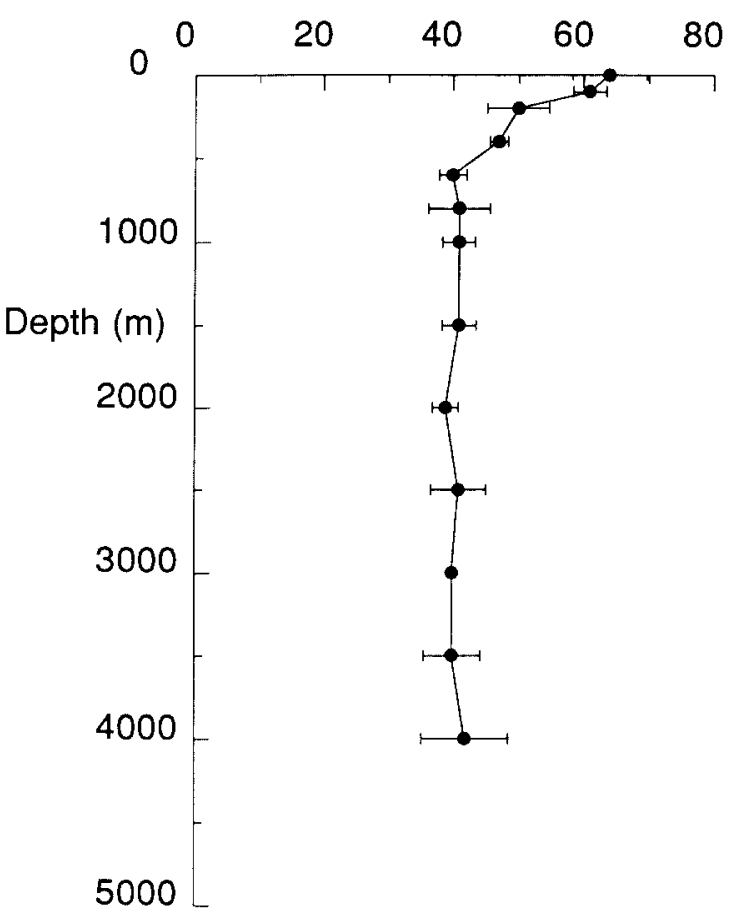

Fig. 2: A composite depth profile from the Equatorial Pacific. Each sample point is the average of the independent analyses by 3 HTC instruments and the persulfate oxidation method; brackets around the points are $\pm S D$.

\section{The Next Step}

The next step is another broad community interlaboratory comparison. This will be similar to the Seattle workshop exercise but will take advantage of what has been learned since. The purpose of this comparison will be to see how well any laboratory can get agreement with DOC analysis using rigorous protocol, blanks, and standards. Participants will have the opportunity to see results from the two recent comparisons mentioned previously and to do trial runs of blanks and standards before committing to the "blind" samples that are to be analyzed. Analysts with any HTC or WCO method are encouraged to participate.

For the trial runs, a blank of very close to zero carbon water will be used as well as two reference standards. Anyone interested can receive these samples and report and discuss results before agreeing to participate in the intercalibration exercise. Those who do agree to participate will be sent blanks, standards, and a set of unknown samples. These analysts will be asked to submit all raw data to me and to be willing to be identified with their data. We hope to distribute the trial run samples during the period of August through October with results due by December. The regular comparison samples will be distributed in January with results due by March 1994.
Anyone interested in participating in this exercise should contact Jonathan Sharp at College of Marine Studies, University of Delaware, Lewes, DE 19958, USA, by Omnet at J. SHARP, by Internet at jsharp@brahms.udel.edu, or FAX at 302$645-4007$.

\section{Acknowledgements}

Thanks to the analysts who worked on this activity for the past one and one-half years; specifically Ron Benner, Lenore Bennett, Craig Carlson, Rachael Dow, Steve Fitzwater, Ed Peltzer, and Luis Tupas. The analysts, Hugh Ducklow, and the late John Martin have been extremely patient and cooperative with someone looking over shoulders and providing unsolicited advice and sometimes officious comments. Through what has been a truly cooperative effort, we were able to make considerable progress toward establishing DOC analysis as a routine activity. Thanks to the DOM Steering Committee of John Hedges, Cindy Lee, Chuck Hopkinson, and Tony Knap for advice and discussion on the intercalibration efforts. Several individuals, not a part of the intercalibration exercises, aided with samples, analyses, and advice. Of those, Brian Fry, Bernie Bernard, Mark Alperin, and Wenhao Chen were especially helpful. Lenore Bennett and Hugh Macintyre helped with the graphics, and John Hedges and Ed Peltzer provided

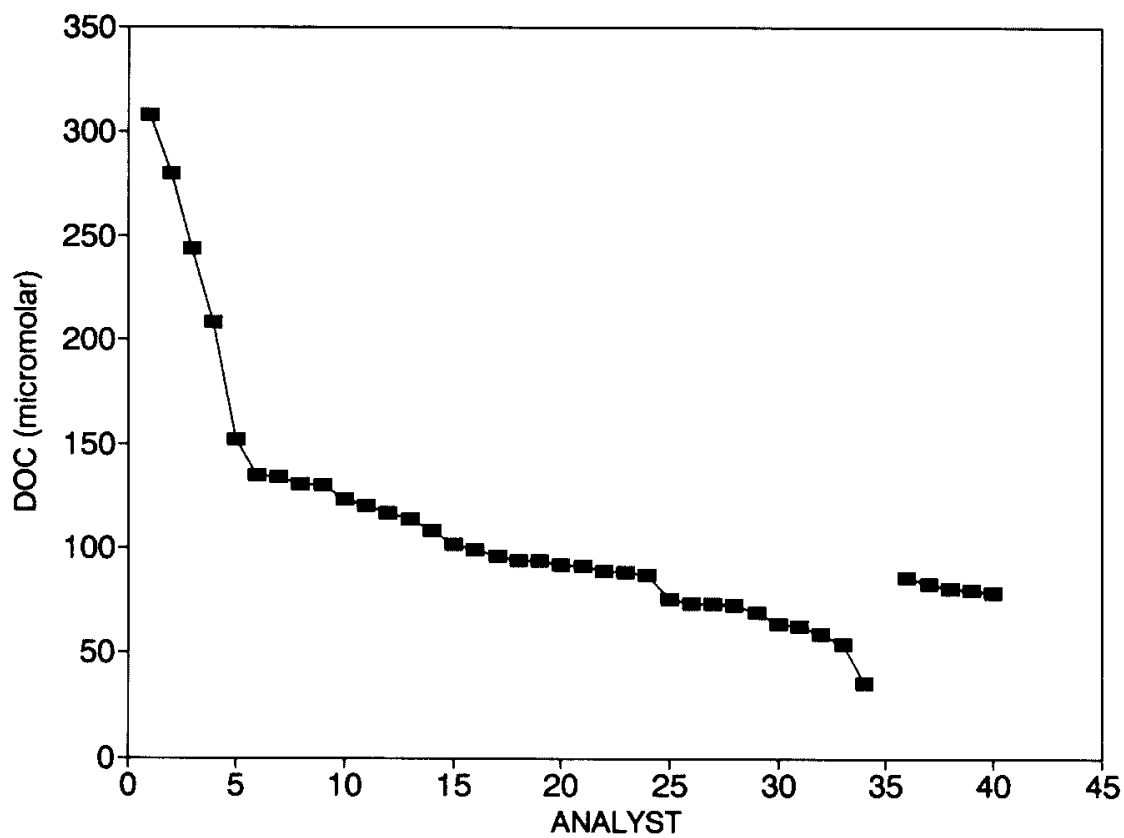

Fig. 3: Data from all analyses of the surface Pacific Ocean sample from the Seattle DOM workshop (analysts 1-34) and from an Equatorial Pacific interlaboratory comparison surface sample (analysts 36-40). Seattle data are from Hedges et al., 1993; all EqPac intercalibration data are presented in Sharp et al.. the manuscript in preparation mentioned in text. The Seattle Workshop data are from a variety of different methods without consistent blank correction. The EqPac data are from 4 HTC analyses and 1 persulfate oxidation analysis with uniform blank correction and reference standards. 
valuable advice on an earlier version of this manuscript. Support for this activity came from NSF grant OCE 92-00970 to J.H. Sharp and the NSF grant for the U.S. JGOFS Steering Committee (OCE 92-08261 to H.D. Livingston). I would like to dedicate this article to John H. Martin who applied the same vision. innovation, and courage to his pursuit of DOC analyses that he did to other oceanographic research areas and life in general.

\section{References}

Bauer, J.E., P.M. Williams and E.R.M. Druffel, 1992: ${ }^{14} \mathrm{C}$ activity of dissolved organic carbon fractions in the northcentral Pacific and Sargasso Sea. Nature 357, 667-670.

Benner, R. and J.I. Hedges, 1993: A test of the accuracy of freshwater DOC measurements by high temperature catalytic oxidation and UV-promoted persulfate oxidation. Mar. Chem. 41, 161-165.

Benner, R., J.D. Pakulski, M. McCarthy, J.I. Hedges and P.G. Hatcher, 1992: Bulk chemical characterization of dissolved organic matter in the ocean. Science 255, 1561-1564.

Benner, R. and M. Strom, 1993: A critical evaluation of the analytical blank associated with DOC measurements by hightemperature catalytic oxidation. Mar. Chem. 41, 153-160.

Chen, W.. 1992: The measurement, production, and degradation of marine dissolved organic matter. Ph.D. dissertation, Dalhousie University.

Druffel, E.R.M., P.M. Williams and Y. Suzuki, 1989: Concentrations and radiocarbon signatures of dissolved organic carbon in the Pacific Ocean. Geophvs. Res. Lett. 16, 991-994. P.M. Williams, J.E. Bauer and J.R. Ertel, 1992: Cycling of dissolved and particulate organic matter in the open ocean. J. Geophys. Res. 97, 15639-15659.

Fry, B.. S. Saupe, M. Hullar and B.J. Peterson, 1993: Platinum-catalyzed combustion of DOC in sealed tubes for stable isotopic analysis. Mar. Chem. 41, 187-193.

Gershey, R.M., M.D. Mckinnon, P.J.LeB. Williams and R.M. Moore, 1979: Comparison of three oxidation methods used for the analysis of dissolved organic carbon in seawater. Mar. Chem. 7, 289-306.

Gordon, D.C. and W.H. Sutcliffe, 1973: A new dry combustion method for the simultaneous determination of total organic carbon and nitrogen in seawater. Mar. Chem. l, 231-244.

Hedges, J.I. and B.A. Bergamaschi, 1992: Seawater carbon measurement. Nature 359, 202.

B.A. Bergamaschi and R. Benner, 1993: Comparative analyses of DOC and DON in natural waters. Mar. Chem. 41, 121-134.

Jackson, G.A., 1988: Implications of high dissolved organic matter concentrations for oceanic properties and processes. Oceanography J, 28-33.

Kaplan, L.A., 1992: Comparison of high-temperature and persulfate oxidation methods for determination of dissolved organic carbon in fresh waters. Limnol. Oceanogr. 37, $1119-1125$
Kepkay, P.E. and M.L. Wells, 1992: Dissolved organic carbon in North Atlantic surface waters. Mar. Ecol. Prog. Ser. $80,275-283$

Martin. J.H. and S.E. Fitzwater, 1992: Dissolved organic carbon in the Atlantic, Southern, and Pacific Oceans. $\mathrm{Na}$. ture 356, 699-700.

McKinnon, M.D.. 1978: A dry oxidation method for the analysis of the TOC in seawater. Mar. Chem. 7, 17-37.

Menzel, D.W. and J.H. Ryther, 1970: Distribution and cycling of organic matter in the oceans. In: Organic Matter in Natural Waters, D.W. Hood, ed. 31-54.

and R.F. Vacarro, 1964: The measurements of dissolved organic and particulate carbon in seawater. Limnol. Oceanogr. 9, 138-142.

Ogawa, H. and N. Ogura, 1992: Comparison of two methods for measuring dissolved organic carbon in sea water. Nature 356, 696-698.

Peltzer, E.T., and P.G. Brewer, 1993: Some practical aspects of measuring DOC-Sampling artifacts and analytical problems with marine samples. Mar. Chem. 4I. 243-252.

Ridal, J.J., 1992: A study of the production and measurement of dissolved organic matter in seawater. Ph.D. dissertation, Dalhousie University. $209 \mathrm{pp}$.

Sharp, J.H., 1973: Total organic carbon in seawater-comparison of measurements using persulfate oxidation and high temperature combustion. Mar. Chem. I, 211-229.

R. Bennet, C.A. Carlson, R. Dow, and S.E. Fitzwater, 1994: Re-evaluation of high temperature combustion instruments for measurement of dissolved carbon in seawater. Limnol. Oceanogr. (In press).

Y. Suzuki, and W.L. Munday, 1993: A comparison of dissolved organic carbon in North Atlantic Ocean nearshore waters by high temperature combustion and wet chemical oxidation. Mar. Chem. 41, 253-259.

Skopintsev, B.A.. S.N. Timofeeva. and O.A. Vershinina, 1966: Organic carbon in the equatorial and southern Atlantic and Mediterranean. Okeanologiva 6, 201-210.

Sugimura, Y. and Y. Suzuki, 1988: A high temperature catalytic oxidation method for non-volatile dissolved organic carbon in seawater by direct injection of a liquid sample. Mar. Chem. 24, 105-131.

Suzuki, Y, 1993. On the measurement of DOC and DON in seawater. Mar. Chem. 41, 287-288.

Y. Sugimura, and T. Ito. 1985: A catalytic oxidation method for the determination of total nitrogen dissolved in seawater. Mar. Chem. 16, 83-97.

Tanoue, E.. 1992: Vertical distribution of dissolved organic carbon in the North Pacific as determined by high temperature catalytic oxidation method. Earth, Planetary Sci. Lttrs. 111, 201-216.

Williams, P.M., 1992: Measurement of dissolved organic carbon and nitrogen in natural waters. Oceanography 5, 107-116. and E.R.M. Druffel, 1988: Dissolved organic matter in the ocean: Comments on the controversy. Oceanography $1,14-17$. 